\title{
Determination of rational transformation coefficients of transformers distribution networks
}

\author{
Dauren Akhmetbayev ${ }^{1 *}$, Arman Akhmetbayev ${ }^{2}$ Aigerim Aidarova $^{3}$ \\ ${ }^{1}$ ENU named by L.N. Gumilev, Astana, Kazakhstan \\ ${ }^{2}$ Directorate of Information Systems of JSC "Kazakhtelecom", Almaty, Kazakhstan \\ ${ }^{3}$ OJSC «Severelectro», Bishkek, Kyrgyzstan
}

\begin{abstract}
The thesis proposed a systematic approach to the determination of rational coefficients transformation of transformers distribution networks. Analytic expressions are obtained for transformation coefficient of transformer from the point of view of synthesis of distribution networks in the field of permissible voltage regimes. The Newton-Raphson method was used to solve nonlinear equations for transformation coefficients. This eliminates the need for calculation of steady distribution network modes.
\end{abstract}

\section{Synthesis formalization for the simplest networks}

For the sake of clarity and simplicity of presentation, we consider the stationary mode of the distribution network shown in Pic. 1, a.

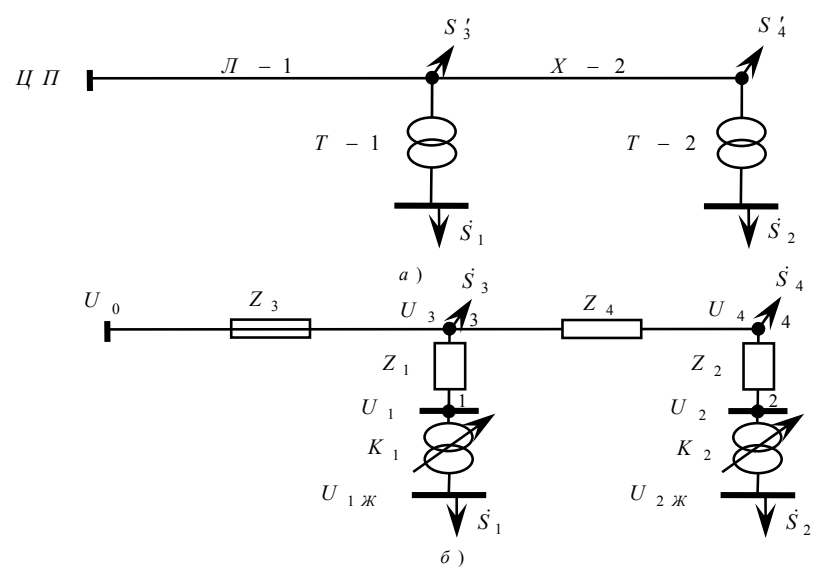

a-principle scheme; $\sigma$ - replacement scheme

Fig. 1. Electrical network scheme

- $Z_{1}, Z_{2}$ - complex resistance of transformers

- $Z_{3}, Z_{4}$ - line resistance of power transmission;

- $\underline{\dot{S}}_{1}, \underline{S}_{2}, \dot{S}_{3}, \dot{S}_{4}$ - calculating power of nodal loads;

- $K_{1}, K_{2}$ - transformation coefficients of ideal transformers
Assume that providing the required voltage quality changes achieved transformation ratio transformers with tap changer on the basis of a counter voltage regulation. Adjusting range taps of transformers is considered sufficient to provide the required voltage levels. Consequently, the desired value of the stress on the tires reducing substation considered as given quantities.

The resulted voltages of the secondary windings of transformers with adjustable transformation coefficients, in accordance with the scheme of Fig. $6.3 \mathrm{~b}$, are determined by the expressions:

$$
\begin{aligned}
& \dot{U}_{1}=U_{0}-\frac{\sum_{j=1}^{4} \underline{C}_{1 j}^{t} \underline{Z}_{j} \underline{C}_{j 1} \hat{S}_{1}}{\hat{U}_{1}}-\frac{\sum_{j=1}^{4} \underline{C}_{1 j}^{t} \underline{Z}_{j} \underline{C}_{j 2} \hat{S}_{2}}{\hat{U}_{2}}- \\
& -\frac{\sum_{j=1}^{4} \underline{C}_{1 j}^{t} \underline{Z}_{j} \underline{C}_{j 3} \hat{S}_{3}}{\hat{U}_{3}}-\frac{\sum_{j=1}^{4} \underline{C}_{1 j}^{t} \underline{Z}_{j} \underline{C}_{j 4} \hat{S}_{4}}{\hat{U}_{4}} \\
& \dot{U}_{2}=U_{0}-\frac{\sum_{j=1}^{4} \underline{C}_{2 j}^{t} \underline{Z}_{j} \underline{C}_{j 1} \hat{S}_{1}}{\hat{U}_{1}}-\frac{\sum_{j=1}^{4} \underline{C}_{2 j}^{t} \underline{Z}_{j} \underline{C}_{j 2} \hat{S}_{2}}{\hat{U}_{2}}- \\
& -\frac{\sum_{j=1}^{4} \underline{C}_{2 j}^{t} \underline{Z}_{j} \underline{C}_{j 3} \hat{S}_{3}}{\hat{U}_{3}}-\frac{\sum_{j=1}^{4} \underline{C}_{2 j}^{t} \underline{Z}_{j} \underline{C}_{j 4} \hat{S}_{4}}{\hat{U}_{4}}
\end{aligned}
$$

where, $\sum_{j=1}^{4} \underline{C}_{1 j}^{t} \underline{Z}_{j} \underline{C}_{j 1}, \sum_{j=1}^{4} \underline{C}_{1 j}^{t} \underline{Z}_{j} \underline{C}_{j 2}$ , ... $\sum_{j=1}^{4} \underline{C}_{2 j}^{t} \underline{Z}_{j} \underline{C}_{j 1}, \sum_{j=1}^{4} \underline{C}_{1 j}^{t} \underline{Z}_{j} \underline{C}_{j 2^{-}}$partial systemic functions of resistance relatively to the first and second nodes.

Without taking into account the influence of the phases of the node voltages on the magnitude of the driving currents, the system (1) is written in the form:

\footnotetext{
*Corresponding author: axmetbaev46@mail.ru
} 


$$
\begin{aligned}
& \dot{U}_{1}=U_{0}-\frac{\sum_{j=1}^{4} \underline{C}_{1 j}^{t} \underline{Z}_{j} \underline{C}_{j 1} \hat{S}_{1}}{U_{1}}-\frac{\sum_{j=1}^{4} \underline{C}_{1 j}^{t} \underline{Z}_{j} \underline{C}_{j 2} \hat{S}_{2}}{U_{2}}- \\
& -\frac{\sum_{j=1}^{4} \underline{C}_{1 j}^{t} \underline{Z}_{j} \underline{C}_{j 3} \hat{S}_{3}}{U_{3}}-\frac{\sum_{j=1}^{4} \underline{C}_{1 j}^{t} \underline{Z}_{j} \underline{C}_{j 4} \hat{S}_{4}}{U_{4}} \\
& \dot{U}_{2}=U_{0}-\frac{\sum_{j=1}^{4} \underline{C}_{2 j}^{t} \underline{Z}_{j} \underline{C}_{j 1} \hat{S}_{1}}{U_{1}}-\frac{\sum_{j=1}^{4} \underline{C}_{2 j}^{t} \underline{Z}_{j} \underline{C}_{j 2} \hat{S}_{2}}{U_{2}}- \\
& -\frac{\sum_{j=1}^{4} \underline{C}_{2 j}^{t} \underline{Z}_{j} \underline{C}_{j 3} \hat{S}_{3}}{U_{3}}-\frac{\sum_{j=1}^{4} \underline{C}_{2 j}^{t} \underline{Z}_{j} \underline{C}_{j 4} \hat{S}_{4}}{U_{4}}
\end{aligned}
$$

Known values of node voltages are such that the system (2) describes the stationary condition of the electrical network within the allowable regimes by voltage.

Desired voltages on tires $6-10 \mathrm{kV}$ of reducing substations are provided by voltage regulation by changing the coefficient transformation of transformers. It is known that the selection of the regulating branches of the transformers is carried out individually for each substation according to the results of calculation of the stationary regime. In this case, the mutual influence of the regulating branches can not be taken into account when choosing the most suitable modes within the range of admissible regimes. The most suitable mode can be found in the system approach to the determination of the regulating branches of all transformers of the lowering substations.

Let the necessary expedient regime of the voltage of the network in question be ensured by changing the transformation ratios of the transformers.

Then the system of equations (2), taking into account the transformation coefficients of ideal transformers, can be written as follows:

$$
\begin{aligned}
& \dot{U}_{1}=U_{0}-\frac{\sum_{j=1}^{4} \underline{C}_{1 j}^{t} \underline{Z}_{j} \underline{C}_{j 1} \hat{S}_{1}}{K_{1} U_{1} \text { }}-\frac{\sum_{j=1}^{4} \underline{C}_{1 j}^{t} \underline{Z}_{j} \underline{C}_{j 2} \hat{S}_{2}}{K_{2} U_{2} \text { K }}- \\
& -\frac{\sum_{j=1}^{4} \underline{C}_{1 j}^{t} \underline{Z}_{j} \underline{C}_{j 3} \hat{S}_{3}}{U_{3}}-\frac{\sum_{j=1}^{4} \underline{C}_{1 j}^{t} \underline{Z}_{j} \underline{C}_{j 4} \hat{S}_{4}}{U_{4}} \\
& \dot{U}_{2}=U_{0}-\frac{\sum_{j=1}^{4} \underline{C}_{2 j}^{t} \underline{Z}_{j} \underline{C}_{j 1} \hat{S}_{1}}{K_{1} U_{1}{ }_{1}}-\frac{\sum_{j=1}^{4} \underline{C}_{2 j}^{t} \underline{Z}_{j} \underline{C}_{j 2} \hat{S}_{2}}{K_{2} U_{2}{ }_{2}}- \\
& -\frac{\sum_{j=1}^{4} \underline{C}_{2 j}^{t} \underline{Z}_{j} \underline{C}_{j 3} \hat{S}_{3}}{U_{3}}-\frac{\sum_{j=1}^{4} \underline{C}_{2 j}^{t} \underline{Z}_{j} \underline{C}_{j 4} \hat{S}_{4}}{U_{4}}
\end{aligned}
$$

Taking into account $U_{1}=U_{1 \%} / K_{1} ; U_{2}=U_{2 \%} / K_{2}$ and the values of the distribution coefficients, the system (3) can be written in the form:

$$
\left.\begin{array}{l}
U_{\Delta 11} K_{1}^{2}+U_{\Delta 12} K_{2} K_{1}-\left(U_{0}-U_{\Delta 13}-U_{\Delta 14}\right) K_{1}+U_{1 \%}=0 \\
U_{\Delta 21} K_{1} K_{2}+U_{\Delta 12} K_{2}^{2}-\left(U_{0}-U_{\Delta 23}-U_{\Delta 24}\right) K_{2}+U_{2 \nVdash}=0
\end{array}\right\}
$$

where the coefficients of the unknowns for the scheme under consideration are equal to:

$$
\begin{aligned}
& U_{\Delta 11}=\sqrt{\left(\frac{P_{1}\left(R_{1}+R_{3}\right)+Q_{1}\left(X_{1}+X_{3}\right)}{U_{1 \%}}\right)^{2}+\left(\frac{P_{1}\left(X_{1}+X_{3}\right)-Q_{1}\left(R_{1}+R_{3}\right)}{U_{1 \%}}\right)^{2}} \\
& U_{\Delta 12}=\sqrt{\left(\frac{P_{2} R_{3}+Q_{2} X_{3}}{U_{2}{ }_{2}}\right)^{2}+\left(\frac{P_{2} X_{3}-Q_{2} R_{3}}{U_{2}{ }_{2}}\right)^{2}} \\
& U_{\Delta 13}=\sqrt{\left(\frac{P_{3} R_{3}+Q_{3} X_{3}}{U_{3}}\right)^{2}+\left(\frac{P_{3} X_{3}-Q_{3} R_{3}}{U_{3}}\right)^{2}} \\
& U_{\Delta 14}=\sqrt{\left(\frac{P_{4} R_{3}+Q_{4} X_{3}}{U_{4}}\right)^{2}+\left(\frac{P_{4} X_{3}-Q_{4} R_{3}}{U_{4}}\right)^{2}} \\
& U_{\Delta 21}=\sqrt{\left(\frac{P_{1} R_{3}+Q_{1} X_{3}}{U_{1}}\right)^{2}+\left(\frac{P_{1} X_{3}-Q_{1} R_{3}}{U_{1}}\right)^{2}} \\
& U_{\Delta 2}=\sqrt{\left(\frac{P_{2}\left(R_{2}+R_{3}+R_{4}\right)+Q_{2}\left(X_{2}+X_{3}+X_{4}\right)}{U_{2 Ж}}\right)^{2}+\left(\frac{P_{2}\left(X_{2}+X_{3}+X_{4}\right)-Q_{2}\left(R_{2}+R_{3}+R_{4}\right)}{U_{2 Ж}}\right)^{2}} ; \\
& U_{\Delta 23}=\sqrt{\left(\frac{P_{3} R_{3}+Q_{3} X_{3}}{U_{3}}\right)^{2}+\left(\frac{P_{3} X_{3}-Q_{3} R_{3}}{U_{3}}\right)^{2}} \\
& U_{\Delta 24}=\sqrt{\left(\frac{P_{4}\left(R_{3}+R_{4}\right)+Q_{4}\left(X_{3}+X_{4}\right)}{U_{4}}\right)^{2}+\left(\frac{P_{4}\left(X_{3}+X_{4}\right)-Q_{4}\left(R_{3}+R_{4}\right)}{U_{4}}\right)^{2}}
\end{aligned}
$$

\begin{tabular}{|c|c|}
\hline Direct method & Classical method \\
\hline \multicolumn{2}{|c|}{ Mode of maximum loads } \\
\hline $\mathrm{K}_{2}=0,3217$ & $\mathrm{U}_{\text {жел }}=10,5 \mathrm{kV}$ \\
\hline $\mathrm{K}_{1}=0,3123$ & $\mathrm{U}_{\text {отв }}=\mathrm{U}_{\mathrm{H}}-\mathrm{n} \times 1,5 \%$ \\
\hline $\mathrm{U}_{2}=34,19 \mathrm{kV}$ & $\mathrm{U}_{\text {отв } 2}=34,47 \mathrm{kV}$ \\
\hline $\mathrm{U}_{1}=35,22 \mathrm{kV}$ & $\mathrm{U}_{\text {отв } 1}=35,52 \mathrm{kV}$ \\
\hline \multicolumn{2}{|c|}{ Minimum mode } \\
\hline $\mathrm{K}_{2}=0,279$ & $\mathrm{U}_{\text {жел }}=10,0 \mathrm{kV}$ \\
\hline $\mathrm{K}_{1}=0,277$ & $\mathrm{U}_{\text {отв } 2}=39,4 \mathrm{kV}$ \\
\hline $\mathrm{U}_{2}=39,42 \mathrm{kV}$ & $\mathrm{U}_{\text {отв } 1}=39,78 \mathrm{kV}$ \\
\hline $\mathrm{U}_{1}=39,71 \mathrm{kV}$ & \\
\hline
\end{tabular}

As an illustration, calculations are made by the proposed and conventional methods for the circuit in Fig. 1 with the specified parameters of the transformers, line and load:

$$
\begin{aligned}
& \square P_{\kappa}=45,5 \kappa B A, \square P_{x}=9,4 \kappa B m, U_{\kappa}=7,5 \%, I_{x}=0,9 \%, \\
& \pm 6 \times 1,5 \%, U_{H}=11 \kappa B
\end{aligned}
$$$$
Л_{1}-2 X A C-70, Л_{2}-2 X A C-35, \mathrm{~L}_{1}=\mathrm{L}_{2}=18 \text { км., } \mathrm{S}_{1}=\mathrm{S}_{2}=3,5+\mathrm{j}
$$

2,5 MBA.

Design parameters: transformers $Z_{1}=$ $\mathrm{Z}_{2}=1,6+\mathrm{j} 16,1$ OM,

line $-Z_{3}=4,05+j 3,78$ Ом, $Z_{4}=8,19+j 4$ Ом.

Calculations of non-linear equations were carried out by the Newton-Raphson method in the MATLAB environment, and the working mode of the network by the two-stage method according to the data of the beginning. The results are shown in Table 1 .

Table 1. Results of calculations

As can be seen from the table, the results obtained by different methods give good coincidences, which allows us to conclude that the 
proposed method can be used to solve both operational and design tasks.

\section{Synthesis of distribution networks of complex structure}

Assume that the desired voltage busbar transformer substations included first $\mathrm{n}$ complex electrical circuit nodes network provided by the transformation ratio transformers changes. Then a nonlinear matrix equation (8.8), after simple transformation can be written in the form of voltage balance, for example $\mathrm{i}$-th node:

$$
\underline{\dot{U}}_{i \varkappa c}=\underline{\dot{U}}_{\Delta i 1} K_{i} K_{1}+\underline{\dot{U}}_{\Delta i 2} K_{i} K_{2}+\ldots+\underline{\dot{U}}_{\Delta i n} K_{i} K_{n}+\left(\sum_{i=n+1}^{N} \underline{\dot{U}}_{\Delta i j}+\underline{\dot{U}}_{0}\right) K_{i}
$$

where

$$
\underline{\dot{\mathbf{U}}}_{\Delta i 1}=\sum_{j=1}^{m} \underline{\mathbf{C}}_{i j}^{t} \underline{\mathbf{Z}}_{j} \underline{\mathbf{C}}_{j 1} \dot{\mathbf{U}}_{1 \%}^{-1} \dot{\mathbf{\mathbf { S }}}_{1},
$$

$\underline{\mathbf{U}}_{\Delta i n}=\sum_{j=1}^{m} \underline{\mathbf{C}}_{i j}^{t} \underline{\mathbf{Z}}_{j} \underline{\mathbf{C}}_{j n} \dot{\mathbf{U}}_{n \varkappa \kappa}^{-1} \underline{\mathbf{S}}_{n}$

- partial nodal voltage

reduces of the $\mathrm{i}$-th node.

$\dot{U}_{i ж}$ - complex desired voltage of the i-th node;

$\underline{\dot{U}}_{0}$ - the voltage of the basic node;

$K_{1}, K_{2}, \ldots, K_{n}$-transformation coefficients of transformers;

$N$ - number of independent nodes.

The dependent variables in equation (4) are the node voltage phases $(\delta)$ and the transformation coefficients transformer $(\mathrm{K})$.

The system of nonlinear equations for the balance of the stress of the node under consideration concerning the variables $(\delta, K)$ can be obtained from (5) by selecting the real and imaginary parts in the form:

$$
\left.\begin{array}{l}
\omega_{U_{i}^{\prime}}(\delta, K)=-U_{i \varkappa \mathcal{C}} \cos \delta_{i}+\sum_{j=1}^{n} U_{\Delta i j} K_{i} K_{j} \cos \left(\delta_{j}-\varphi_{j}+\psi_{i j}\right)+ \\
+\left(\sum_{j=n+1}^{N} U_{\Delta i j} \cos \left(\delta_{j}-\varphi_{j}+\psi_{i j}\right)+U_{0}\right) K_{i} \\
{\underline{\underline{\dot{U}_{i}^{\prime \prime}}}}(\delta, K)=-U_{i \varkappa \mathcal{C}} \sin \delta_{i}+\sum_{j=1}^{n} U_{\Delta i j} K_{i} K_{j} \sin \left(\delta_{j}-\varphi_{j}+\psi_{i j}\right)+ \\
+\left(\sum_{j=n+1}^{N} U_{\Delta i j} \sin \left(\delta_{j}-\varphi_{j}+\psi_{i j}\right)\right) K_{i}
\end{array}\right\}
$$

Where:

$U_{i}^{\prime}, U_{i}^{\prime \prime}$ are the real and imaginary parts of the voltage;

$U_{\Delta i j}$ - module of partial nodal voltage drop;

$\varphi_{j}$ - phase shift of the load power of the j-th node; $\psi_{i j}$ - angle of mutual complex nodal resistance.

It is seen from (6) that a system of $2 n$ equations with $2 \mathrm{n}$ unknowns is compiled, the solution of which can be obtained by the Newton method. The Jacobi matrix is not degenerate and can be written in block form in the form:

$$
\frac{\partial \mathbf{W}_{U}(x)}{\partial x}=\left\|\begin{array}{ll}
\frac{\partial \omega_{v^{\prime}}(\delta, K)}{\partial \delta} & \frac{\partial \omega_{v^{\prime}}(\delta, K)}{\partial K} \\
\frac{\partial \omega_{v^{\prime \prime}}(\delta, K)}{\partial \delta} & \frac{\partial \omega_{v^{\prime \prime}}(\delta, K)}{\partial K}
\end{array}\right\|
$$

The partial derivatives of the i-th row of each block matrix can be calculated as follows:

$\frac{\partial \omega_{v^{\prime} i}(\delta, K)}{\partial \delta}=U_{i \rightsquigarrow c} \sin \delta_{i}-U_{\Delta i i} K_{i}^{2} \sin \left(\delta_{i}-\varphi_{i}+\psi_{i i}\right)-\sum_{\substack{j=1 \\ j \neq i}}^{n} U_{\Delta i j} K_{i} K_{j} \sin \left(\delta_{j}-\varphi_{j}+\psi_{i j}\right) ;$

$\frac{\partial \omega_{v^{\prime} i}(\delta, K)}{\partial K}=2 U_{\Delta i i} K_{i} \cos \left(\delta_{i}-\varphi_{i}+\psi_{i i}\right)+\sum_{j=n+1}^{n} U_{\Delta i j} \cos \left(\delta_{j}-\varphi_{j}+\psi_{i j}\right)+U_{0}+$

$+\sum_{\substack{j=1 \\ j=i}}^{N} U_{\Delta i j} K_{i} \cos \left(\delta_{j}-\varphi_{j}+\psi_{i j}\right)$

$\frac{\partial \omega_{v^{\prime \prime} i}(\delta, K)}{\partial \delta}=-U_{i \varkappa c} \cos \delta_{i}-U_{\Delta i i} K_{i}^{2} \cos \left(\delta_{i}-\varphi_{i}+\psi_{i i}\right)+\sum_{j=1}^{n} U_{\Delta i j} K_{i} K_{j} \cos \left(\delta_{j}-\varphi_{j}+\psi_{i j}\right)$

$\frac{\partial \omega_{v^{\prime \prime} i}(\delta, K)}{\partial K}=2 U_{\Delta i i} K_{i}+\sum_{j=n+1}^{N} U_{\Delta i j} \sin \left(\delta_{j}-\varphi_{j}+\psi_{i j}\right)+\sum_{\substack{j=1 \\ j \neq i}}^{n} U_{\Delta i j} K_{i} \sin \left(\delta_{j}-\varphi_{j}+\psi_{i j}\right)$.

If we neglect the influence of the phases of the node voltages on the values of the driving currents, then the system (6.32) can be written in the form:

$$
\left.\begin{array}{l}
\omega_{\underline{\underline{U}}_{i}^{\prime}}(\delta, K)=-U_{i \varkappa \mathcal{K}} \cos \delta_{i}+\sum_{j=1}^{n} U_{\Delta i j} K_{i} K_{j} \cos \left(-\varphi_{j}+\psi_{i j}\right)+ \\
+\left(\sum_{j=n+1}^{N} U_{\Delta i j} \cos \left(-\varphi_{j}+\psi_{i j}\right)+U_{0}\right) K_{i} \\
\omega_{\underline{U}_{i}^{\prime \prime}}(\delta, K)=-U_{i \varkappa c} \sin \delta_{i}+\sum_{j=1}^{n} U_{\Delta i j} K_{i} K_{j} \sin \left(-\varphi_{j}+\psi_{i j}\right)+ \\
+\left(\sum_{j=n+1}^{N} U_{\Delta i j} \sin \left(-\varphi_{j}+\psi_{i j}\right) K_{i}\right.
\end{array}\right\}
$$

Then the partial derivatives of the matrix are defined as:

$\frac{\partial \omega_{v^{\prime} i}(\delta, K)}{\partial \delta}=U_{i \varkappa c} \sin \delta_{i} ;$

$\frac{\partial \omega_{v^{\prime} i}(\delta, K)}{\partial K}=2 U_{\Delta i i} K_{i} \cos \left(-\varphi_{i}+\psi_{i i}\right)+\sum_{j=n+1}^{N} U_{\Delta i j} \cos \left(-\varphi_{j}+\psi_{i j}\right)+U_{0}+$

$+\sum_{j=1}^{n} U_{\Delta i j} K_{i} \cos \left(-\varphi_{j}+\psi_{i j}\right)$

$\frac{\partial \omega_{v^{\prime \prime} i}(\delta, K)}{\partial \delta}=-U_{i \varkappa} \cos \delta_{i}$ 


$$
\begin{aligned}
& \frac{\partial \omega_{v^{\prime \prime} i}(\delta, K)}{\partial K}=2 U_{\Delta i i} K_{i} \sin \left(-\varphi_{i}+\psi_{i i}\right)+\sum_{j=n+1}^{N} U_{\Delta i j} \sin \left(-\varphi_{j}+\psi_{i j}\right)+ \\
& +\sum_{\substack{j=1 \\
j \neq i}}^{n} U_{\Delta i j} K_{i} \sin \left(-\varphi_{j}+\psi_{i j}\right) .
\end{aligned}
$$

In distribution networks calculation formula for determining the elements of the Jacobian matrix, greatly simplified and reduced amount of calculations performed since the voltage balance equation I - th node can be reduced to the form:

$$
\omega_{\underline{U_{i}}}(K)=-U_{i \jmath c}+\sum_{j=1}^{n} U_{\Delta i j} K_{i} K_{j} \cos \left(-\varphi_{j}+\psi_{i j}\right)+\left(\sum_{j=n+1}^{N} U_{\Delta i j} \cos \left(-\varphi_{j}+\psi_{i j}\right)+U_{0}\right) K_{i}
$$

which can easily be written in the matrix form as follows:

$$
\mathbf{K}_{д} \mathbf{U}_{\Delta} \mathbf{K}+\left(\mathbf{U}_{\Delta \Sigma}+\mathbf{U}_{0} \mathbf{E}\right) \mathbf{K}-\mathbf{U}_{\varkappa}=0
$$

$\mathbf{U}_{\Delta}$ - square matrix of nodal voltage drops from load currents, powered from transformer buses;

$\mathbf{U}_{\Delta \Sigma}$ - column matrix of nodal voltage drops from load currents without transformer links;

$\mathbf{U}_{\mathscr{A}}$ - column matrix of the desired stresses;

$\mathbf{K}$ - column matrix of transformation coefficients of transformers;

E - the identity matrix.

Given the initial approximations of the transformation coefficients of transformers and expanding in a Taylor series, we can obtain a linear approximation of each nonlinear equation of the system (6.34), for example, the nonlinear approximation of the i-th node has the following form:

$$
\begin{aligned}
& -U_{i j i}+U_{\Delta i 1} \cos \left(-\varphi_{1}+\psi_{i 1}\right) K_{i}^{0} K_{1}^{0}+\ldots+U_{\Delta i i} \cos \left(-\varphi_{i}+\psi_{i i}\right)\left(K_{i}^{0}\right)^{2}+\ldots+ \\
& +U_{\Delta i n} \cos \left(-\varphi_{n}+\psi_{i n}\right) K_{i}^{0} K_{n}^{0}+\left(\sum_{j=n+1}^{N} U_{\Delta i j} \cos \left(-\varphi_{j}+\psi_{i j}\right)+U_{0}\right) K_{i}^{0}+ \\
& +U_{\Delta i 1} \cos \left(-\varphi_{1}+\psi_{i 1}\right) K_{i}^{0} K_{1}^{(1)}+\ldots+\left(2 U_{\Delta i} \cos \left(-\varphi_{i}+\psi_{i i}\right) K_{i}^{0}+\right. \\
& \left.+\sum_{j=n+1}^{N} U_{\Delta i j} \cos \left(-\varphi_{j}+\psi_{i j}\right)+U_{0}\right) \Delta K_{i}^{(1)}+\ldots+U_{\Delta i n} \cos \left(-\varphi_{n}+\psi_{i n}\right) K_{i}^{0} \Delta K_{n}^{(1)}=0
\end{aligned}
$$

We write the system of linearized equations in the matrix form as follows:

$$
W\left(K^{0}\right)+\frac{\partial W\left(K^{0}\right)}{\partial K} \Delta K^{(1)}=0
$$

Where $\Delta K^{(1)}$ is the matrix of corrections of the first iteration of Newton. Each step of the iterative process involves solving the linear system (11) with the subsequent determination of the approximation:

$$
K^{(i+1)}=K^{i}+\Delta K^{(i+1)}
$$

The Newtonian iteration process, in general, can be written in matrix form:

$$
K^{(i+1)}=K^{(i)}-\left[\frac{\partial W\left(K^{i}\right)}{\partial K}\right]^{-1} W\left(K^{i}\right)
$$

Control of convergence is carried out on the vector of residuals:

$$
W_{v}(K) \leq \varepsilon
$$

Where $\varepsilon$ - is a predetermined small value.

From the calculated values of the transformation coefficients, it is easy to find the tap voltage and the regulating stage of each transformer.

\section{Conclusions}

1. A systematic method for calculating the transformer ratios of distribution network transformers has been developed.

2. Nonlinear equations are obtained with respect to transformer transformation coefficients.

3. To solve the problem, the Newton-Raphson method is proposed.

\section{References}

1. V.A. Venikov, Optimization of the regimes of power plants and power systems (M.:Energoatomizdat, 1990). 2. V.I. Idelchik, Calculations and optimization of modes of systems electrical networks (M.: Energoatomizdat, 1988).

3. A.G. Rusina, T.A. Filippova. Modes of power plants and electric power systems. (Novosibirsk: SGTU, 2016).

4. D.S. Ahmetbayev and oth. Development of topological method for calculating current distribution coefficients in complex power networks, Results in Physics, (2017).

5. D.S. Akhmetbayev Topological method of calculating current distribution indices (Worid of Science. Praha:IADE, 2010).

6. D.S. Akhmetbayev Electricity, № 11, (2010). 